\title{
Special issue: Technologies for future high-efficiency industrial silicon wafer solar cells
}

\author{
(C) Higher Education Press and Springer-Verlag Berlin Heidelberg 2016
}

In this special issue we have twelve articles from researchers in Australia and China that discuss various aspects related to technology which could be implemented in the near term in industrial silicon wafer solar cells. In the recent years we have seen the introduction of the passivated emitter rear contact (PERC) solar cell, which was invented at UNSW Australia in the 1980s, in industrial high-volume manufacturing. Hence, it took longer than 30 years from conception to the high-volume manufacturing stage for this solar cell architecture. However, the photovoltaic industry is currently quite different than it was in the 1980s and we expect that new technology can make the 'lab to fab' transition a lot quicker now. This is also partly because many university groups and research institutes now have pilot-line capabilities and can fabricate industry-size solar cells in statistically-relevant batch sizes using industrial production equipment. Approximately half of the articles in this issue deal about technologies which are best described as add-on technologies for the industrial PERC solar cell or discuss aspects of the PERC solar cell in some more detail. The other articles deal with specifics of amorphous-silicon/crystalline-silicon heterojunction solar cells which are expected to obtain an increased market share in the near future.

The first paper by Nampalli et al. from UNSW go into detail into the fundamental recombination parameters of the well-known (but still not fully understood) boronoxygen defect which is the root cause for light-induced degradation observed in p-type PERC solar cells. The second paper from Chen et al. from UNSW deals with optimizing gettering for very lightly P-doped electron collectors. They show that heavily doped and defected regions introduced by laser doping, e.g., used for selective electron collectors, can very effectively getter impurities such as iron resulting in a significant reduction in the defect-related recombination in the bulk regions near these laser-doped features. In the third paper Kim et al. from UNSW look into the effect of the various thermal processes on the final performance of multicrystalline silicon wafer solar cells. They show that the effect of thermal treatments on the solar cell efficiency are not straightforward and highly interconnected. Particularly the gettering and bulk and surface passivation need to be optimized in conjunction to ensure the optimal solar cell performance. The optimal thermal treatment also strongly depends on the quality of the initial multicrystalline silicon wafer e.g., with respect to the impurity concentration. In the fourth paper $\mathrm{Li}$ et al. from UNSW present a nice overview of the optimization of $\mathrm{POCl}_{3}$ diffusion for industrial silicon wafer solar cells. Despite the fact that this process has been the industry standard for decades, there is still a lot of development in this area. In this paper Li et al. particularly focus on the impact of oxidation on the resulting doping profile and its majority and minority carrier properties. They show that the minority carrier recombination can be significantly reduced without detrimental impact on the contact resistance as well as the sheet resistance of the electron collector. The fifth paper by Chen et al. from Shanghai Jiao Tong University present a detailed study on the surface passivation properties of silicon oxide $\left(\mathrm{SiO}_{2}\right)$ films synthesized by liquid phase deposition (LPD) which is a potentially very low cost deposition technology. They show that the $\mathrm{SiO}_{2}$ films show a good level of surface passivation after a post-deposition anneal at $\sim 700^{\circ} \mathrm{C}$. The films were also integrated in industrial silicon wafer solar cells to passive the P-doped electron collector. A $5 \mathrm{~nm}$ LPD-grown $\mathrm{SiO}_{2}$ film was capped with a $\sim 70 \mathrm{~nm}$ silicon nitride and a champion solar cell efficiency of $19.5 \%$ was obtained. In the sixth paper Wenham et al. from UNSW, discuss how advanced hydrogenation can be used to fix crystallographic defects. They demonstrated that the energy conversion efficiency of solar cells fabricated from cast-monocrystalline silicon can be improved by up to $2 \%$ absolute by hydrogen-passivating the dislocation clusters in these solar cells. They also hypnotize that the aluminum back surface field can actually block or trap hydrogen which 
assists in the hydrogen passivation process. In the seventh paper Xie et al. from Zhejiang University show that light-induced degradation can be solved by forward biasing solar cells at elevated temperatures after the co-firing step. They demonstrated that this so-called SCIPT (special current injecting passivation treatment) approach was successful for both standard aluminum back surface field (Al-BSF) as well as PERC solar cells and presents an appealing proposition to the industry. In the eighth paper Chen et al. from UNSW describe a novel way to increase the adhesion strength of plated contact for silicon wafer solar cells. They show that the application of laser-formed anchor points at strategic points of the metallization grid significantly improve the adhesion of the plated metal grid and ensure that these contacts pass the relevant industry standard tests. In the ninth paper Meng et al. from Shanghai Institute of Microsystem and Information Technology, Chinese Academy of Sciences, in collaboration with Longi Silicon Materials Corporation demonstrate over $23 \%$ efficient ntype heterojunction silicon wafer solar cells. The paper presents their detailed optimization with a particular focus on the texturing and passivation. Their champion solar cell efficiency of $23.1 \%$ was obtained on a $100 \mu \mathrm{m}$ industrial Cz-grown wafer. Efficiencies above $22 \%$ have been achieved for wafers taken from the whole $\mathrm{Cz}$ ingot range, demonstrating the industrial potential. In the tenth paper by Zhao et al. from Institute of Electrical Engineering, Chinese Academy of Sciences, a detailed study is presented about the optimisation of the microstructure of the intrinsic amorphous silicon (a-Si: $\mathrm{H}$ ) layer as used in heterojunction silicon wafer solar cells. They showed that relevant information about the passivation quality of the a-Si:H layer could be inferred by the configuration of the bonded hydrogen in the film. Films with a relatively high density of mono-hydrides were found to have a significantly higher level of surface passivation compared to films where hydrogen was bounded in di-hydrides or even poly-hydrides. In the eleventh paper Huang et al. from Nanchang University present a detailed study about the optimisation of amorphous silicon stacks for application in heterojunction silicon wafer solar cells. They show that by carefully optimising the stack, a significant gain in the short-circuit current can be obtained compared to the conventional heterojunction solar cell structure. In the twelfth and final paper Jia et al. from Institute of Microelectronics, Chinese Academy of Sciences present detailed simulations of all-back-contact heterojunction silicon wafer solar cells using advanced technology computer aided design (TCAD). They particularly focus on the role of the front surface field and show that although field-effect passivation is crucial for this layer, the doping density in the n-type doped a-Si:H film should not be too high as it can result in an increased recombination rate.
We sincerely hope you will enjoy reading this special issue and find it an interesting and informative read. We would like to thank all the authors for their submission to this special issue. We are also very grateful to all reviewers for their service and commitment to this journal via their rigorous reviews, timely review, and foremost, to their insightful, constructive comments that further improved the quality of the papers in this special issue.

\section{Guest Editors}

\section{Dr. Wenzhong SHEN}

Professor, Shanghai Jiao Tong University, China

E-mail:wzshen@sjtu.edu.cn

\section{Dr. Bram HOEX}

Senior Lecturer, UNSW Australia, Australia

E-mail: b.hoex@unsw.edu.au

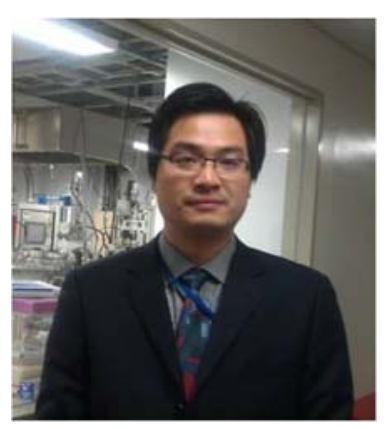

Dr. Wenzhong SHEN is currently a professor of Physics at Shanghai Jiao Tong University. He received his Ph.D. degree in semiconductor physics and semiconductor device from Shanghai Institute of Technical Physics, Chinese Academy of Sciences, in 1995. Since 1999, Dr. Shen has been with Shanghai Jiao Tong University, China, as a full professor in the Department of Physics and Astronomy, where he is currently the director of Institute of Solar Energy, and the leader of the Condensed Matter Spectroscopy \& Optoelectronic Physics Laboratory. He was granted the Changjiang chair professor of National Minister of Education at Shanghai Jiao Tong University in 2000, the Chinese Distinguished Young Scientist Award in 2001, the leader of Changjiang innovation group of National Minister of Education in 2005, and the SNEC distinguished PV scientist award in 2016. Since 2009, Prof. Shen also holds the position of the director of Key Laboratory of Artificial Structures and Quantum Control, Ministry of Education. He was elected in July 2011 and re-elected in July 2015 as the chairman of the Shanghai Solar Energy Society. His main research interests are in the fields of silicon solar cells, nanostructured solar cells and semiconductor quantum electronic devices. He has served as the $\mathrm{PI}$ of 10 projects (including 3 key projects) funded by National Natural Science Foundation of China, authored and co-authored more than 200 papers, 2 monographs, 3 book chapters, and granted 25 patents. 


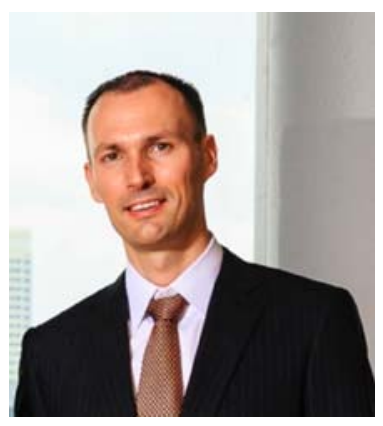

Dr. Bram HOEX completed both a MSc and Ph.D. degree from Eindhoven University of Technology in 2003 and 2008, respectively. From 2008 to 2015 he worked at the Solar Energy Research Institute of Singapore (SERIS) at the National University of Singapore (NUS) as a Group Leader and from 2012 also as Director of the Silicon Materials and Cells Cluster. In 2015 he took up an academic position at the School of Photovoltaic and Renewable Energy Engineering (SPREE) at UNSW Australia and from 2016 he serves as Deputy Head of School (Research). During his career, he has raised over US\$ 15M in competitive research funding of which US\$ 11M as lead Principal Investigator. He has published over 100 journal and conference papers which have been cited over 3400 times. His research focuses on the fabrication and characterization of high-efficiency silicon wafer solar cells. His work has received various international recognitions including the 2008 Solar World Junior Einstein and the 2016 IEEE PVSC Young Professional awards. 